\title{
A Modification of Rician Distribution for SAR Image Modelling
}

Oktay Karakuş $^{* a}$, Ercan E. Kuruoğlu ${ }^{\dagger b}$, and Alin Achim ${ }^{\mathrm{a}}$

${ }^{a}$ Visual Information Lab, University of Bristol, Bristol, U.K.

${ }^{\mathrm{b}}$ Data Science and Information Technology Center, Tsinghua-Berkeley Shenzhen Institute, China

\begin{abstract}
This paper presents a novel statistical model i.e. the Laplace-Rician distribution, for the characterisation of synthetic aperture radar (SAR) images. Since accurate statistical models lead to better results in applications such as target tracking, classification, or despeckling, characterising SAR images of various scenes including urban, sea surface, or agricultural, is essential. The proposed Laplace-Rician model is investigated for SAR images of several frequency bands and various scenes in comparison to state-of-the-art statistical models that include $\mathcal{K}$, Weibull, and Lognormal. The results demonstrate the superior performance and flexibility of the proposed model for all frequency bands and scenes.
\end{abstract}

\section{Keywords}

SAR amplitude, SAR Image, Laplace distribution, Rician distribution, Statistical modelling, Heavy-tailed back-scattering.

\section{Introduction}

Statistical modelling of SAR images plays an essential role in characterising various scenes and underpins applications such as classification $[1,2]$ or denoising $[3,4]$. The literature abounds with numerous statistical models for different SAR scenes, and selecting the most accurate for SAR imagery is essential.

The standard SAR signal model defines the back-scattered SAR signal received by a SAR sensor as a complex signal $R=x+i y$, where $x$ and $y$ are the real and imaginary parts, respectively and follows several assumptions $[5,6]$ :

1. The number of scatterers is large,

2. The scatterers are statistically independent,

3. The instantaneous scattering phases are statistically independent of the amplitudes,

4. The phase is uniformly distributed,

5. The reflectors are relatively small when compared to the illuminated scene,

6. There is no dominating scatterer in the the scene.

In particular, the first two assumptions recall the central limit theorem whereby the real and imaginary parts are jointly Gaussian. Combining with the assumption 6, this leads to the case where $x$ and $y$ are independent and identically distributed (i.i.d.) zero-mean Gaussian random variables. Thence, the amplitude distribution becomes the Rayleigh distribution, the probability density function (pdf) of which is given in (1).

\footnotetext{
*This work was supported by the Engineering and Physical Sciences Research Council (EPSRC) under grant EP/R009260/1 (AssenSAR).

$\dagger$ E.E. Kuruoglu is on leave from ISTI-CNR, Pisa, Italy.
}

Table 1 Probability density functions.

\begin{aligned} Model & Expression \\ \hline Rayleigh $\rightarrow & \frac{r}{\gamma^{2}} e^{-\frac{r^{2}}{2 \gamma^{2}}} \\$ Rician & $\rightarrow \quad \frac{r}{\sigma^{2}} e^{-\frac{r^{2}+\delta^{2}}{2 \sigma^{2}}} \mathcal{I}_{0}\left(\frac{r \delta}{\sigma^{2}}\right) \\ \mathcal{K} & \rightarrow \frac{2}{\gamma \Gamma(\alpha+1)}\left(\frac{r}{2 \gamma}\right)^{\alpha+1} K_{\alpha}\left(\frac{r}{\gamma}\right) \\$ Weibull $\rightarrow & \frac{\alpha}{\gamma}\left(\frac{r}{\gamma}\right)^{\alpha-1} e^{-\left(\frac{r}{\gamma}\right)^{\alpha}} \\$ Lognormal & $\rightarrow \frac{1}{r \gamma \sqrt{2 \pi}} e^{-\frac{(\log r-\mu)^{2}}{2 \gamma^{2}}} \\$ SR [5] & $\rightarrow \quad r \int_{0}^{\infty} s e^{-\gamma s^{\alpha}} \mathcal{J}_{0}(s r) d s \\$ GGR [6] & $\frac{\alpha^{2} r}{4 \gamma^{2} \Gamma^{2}\left(\frac{1}{\alpha}\right)} \int_{0}^{2 \pi} e^{-\frac{|r \cos \theta|^{\alpha}+|r \sin \theta|^{\alpha}}{\gamma^{\alpha}}} d \theta\end{aligned}$

However, in most cases, SAR reflections are nonGaussian/Rayleigh, thus there are numerous statistical models in the literature which were developed to account for non-Rayleigh cases. In cases when the illuminated scene includes a dominating scatterer, where the assumption 6 is no longer valid, $x$ and $y$ become identically and independent non-zero-mean Gaussian random variables, which determines the amplitude distribution to follow the Rician distribution with pdf given in (2).

Furthermore, $\mathcal{K}$ [7], Weibull [8] and Lognormal [9] distributions dominate the literature for modelling several different scenes from urban to agricultural, from land cover to sea surface in SAR imagery. The pdfs of $\mathcal{K}$, Weibull and 
Lognormal models are given in (3), (4) and (5), respectively.

In a previous study, following the non-Gaussian reflections idea, Kuruoglu \& Zerubia [5] have proposed a generalised central limit theorem based statistical model which extends the standard scattering model discussed above by addressing the real and the imaginary parts of the complex backscattered SAR signal as jointly symmetric- $\alpha$-Stable random variables. This model, called the generalised Rayleigh distribution (will be denoted as Stable-Rayleigh, or shortly SR for the rest of the paper) for amplitude SAR images, the pdf of which is given in (6). SR has been shown to be a good choice for urban SAR image modelling in $[5,10]$ and successfully applied to despeckling studies in [3].

Moser et, al, [6] have proposed another generalised statistical model for amplitude SAR modelling, which is similar to SR [5], by assuming the real and imaginary parts of the back-scattered signal to be independent zero-mean generalised Gaussian (GG) random variables, which leads to the generalised Gaussian Rayleigh (GGR) distribution, with the pdf given in (7).

In this paper, we present a novel statistical model, namely the Laplace-Rician distribution for modelling amplitude SAR images. The proposed model is based on the Rician distribution, whereby we assume that the real and imaginary parts of the back-scattered SAR signal are Laplace distributed. Notwithstanding its seldom usage [11, 12], the Rician distribution occurs in cases when a scatterer dominates the SAR scene $[13,14]$, and would characterise several SAR scenes. Combining the Rician idea with the non-Gaussian case via Laplace distribution in the proposed Laplace-Rician model addresses both the non-Rayleigh and heavy-tailed characteristics of amplitude SAR images. The rest of the paper is organised as follows: we present the proposed Laplace Rician model in Section 2. Then, in Section 3, we demonstrate the experimental analysis for various real SAR data, followed by concluding remarks and future work in Section 4.

\section{The Proposed Model}

In this study, we follow the idea of generalising the Rayleigh distribution through heavy-tailed distributions proposed in $[5,6]$, however, this time we apply this to the case when the there are dominant reflectors leading to nonzero mean reflections previously modelled with the Rician distribution.

In particular, we first assume that signal components, $x$ and $y$ are non-zero mean Laplace distributed:

$$
f(u \mid \mu, \gamma)=\frac{1}{2 \gamma} e^{-\left|\frac{u-\mu}{\gamma}\right|},
$$

where $\mu$ is the location parameter, $\gamma$ refers to the scale parameter, and $u$ is either $x$ or $y$. Since the SAR signal components $x$ and $y$ are independent [6], we write the joint pdf as

$$
\begin{aligned}
f(x, y \mid \mu, \gamma) & =f(x \mid \mu, \gamma) f(y \mid \mu, \gamma) \\
& =\frac{1}{4 \gamma^{2}} e^{-\frac{|x-\mu|+|y-\mu|}{\gamma}} .
\end{aligned}
$$

Therefore, by using the identity

$$
f(r, \theta \mid \mu, \gamma)=r f(r \cos \theta, r \sin \theta \mid \mu, \gamma)
$$

we write the joint pdf for the amplitude $(r)$ and phase $(\theta)$ of the back-scattered SAR signal as

$$
f(r, \theta \mid \mu, \gamma)=\frac{r}{4 \gamma^{2}} e^{-\frac{|r \cos \theta-\mu|+|r \sin \theta-\mu|}{\gamma}}
$$

Hence, the corresponding marginal amplitude pdf can be obtained by averaging (12) over $\theta$ and boils down to [15]:

$$
f(r \mid \mu, \gamma)=\frac{r}{4 \gamma^{2}} \int_{0}^{2 \pi} e^{-\frac{|r \cos \theta-\mu|+|r \sin \theta-\mu|}{\gamma}} d \theta
$$

which we call the Laplace-Rician distribution.

\section{$3 \quad$ Results and Discussions}

The proposed method was tested for 33 different SAR images coming from various platforms with frequency bands of $X$ (TerraSAR-X, COSMO-SkyMed and ICEYE), $L$ (ALOS-2) and $C$ (Sentinel-1). Each SAR image corresponds to one type of scene investigate, i.e. urban, agricultural, mountain, land cover and sea surface with and without ships and their wakes. Since we have three sources for $\mathrm{X}$ band SAR imagery as mentioned above, we have more $\mathrm{X}$ band example images in this study, the exact distribution of images in terms of scenes and frequency bands is given in Table 2.

Table 2 Data set details. Distribution of images in terms of scene and frequency bands.

whip: with ships, woShip: without ships.

\begin{tabular}{llll}
\hline \multirow{2}{*}{ Scene } & \multicolumn{3}{c}{ Frequency Band } \\
& X & L & C \\
\hline Urban & 2 & 1 & 2 \\
Agricultural & 1 & 2 & 2 \\
Land & 2 & 1 & 1 \\
Mountain & 2 & 1 & 1 \\
Sea/wShips & 3 & 1 & 1 \\
Sea/woShips & 2 & 1 & 2 \\
Mixed & 2 & 1 & 2 \\
\hline
\end{tabular}

Initially, each utilised SAR image was down-sampled to have a sample size of around 5000. The down-sampling factor was different for each image since images had various sizes. The modelling performance of the LaplaceRician model was compared to state-of-the-art statistical models including $\mathcal{K}$, Rayleigh, Rician, Weibull, lognormal, and GG-Rayleigh of [6].

Model parameters (i.e. $\mu, \gamma, \alpha$ ) for all the statistical models were estimated by using a Bayesian methodology, namely the Metropolis-Hastings algorithm (for details please see [15]). The Kullback-Leibler (KL) divergence and Kolmogorov-Smirnov (KS) score were utilised as statistical significance measures to evaluate the fitting performance of the various distributions. KL values were calculated by assessing the data histogram and the estimated 
pdfs for each statistical model, whilst KS values were calculated by evaluating the empirical and estimated cumulative distribution functions (CDF).

The number of histogram bins for analysis was calculated for each image by using Sturge's method [16]. Finally, the corresponding modelling results are depicted in Figures 1 and 2, and Tables 3 and 4.

When examining the statistical significance values in Table 3 , we can clearly state that the most suitable statistical model for most of the studied scenes is the proposed Laplace-Rician model. Specifically, for 18 out of 33 data sets, the proposed model has both the lowest KL and KS values. Except for 4 out of 33 images utilised in this paper $\left(I_{5}, I_{12}, I_{18}\right.$ and $\left.I_{25}\right)$, the proposed model reaches at least one of the lowest statistical measures of KL and KS. According to the results presented in Table 3, it is hard to conclude on a relationship between model performance and SAR frequency bands.

Examining the Table specifically for the studied scene, it is obvious that the proposed Laplace-Rician model is the best performing model for all the scenes in most of the bands. Weibull, Rician and lognormal models are generally the second best models whilst the remaining fall short in modelling when compared to Laplace-Rician, lognormal, Rician and Weibull for most of the cases.

Particularly for agricultural, mountain and land scenes various models achieved to be the second best model, whilst the Laplace-Rician model achieved at least one lowest statistical significance measures for 11 out of 13 images. For sea scenes, lognormal distribution is generally the second best model for both cases when ships are present or not in the image. For only 1 out of 10 sea surface images $\left(I_{25}\right)$ the proposed model failed to obtain at least one lowest statistical significance measures. For mixed and urban scene, the Laplace-Rician model achieves the best modelling results 9 out of 10 images.

Table 4 presents modelling performance percentages in terms of KL divergence and KS score values. Specifically, from Table 4 we can state that for $60.61 \%$ of images Laplace-Rician is the best model in terms of KL divergence values, whereas $81.82 \%$ in terms of KS score. Lognormal is the second best model in terms of modelling percentages, whilst Rician and Weibull are following the lognormal model with modelling percentages of around 5-10\%.

Figure 1 presents SAR images for six different scenes and their modelling results in logarithmic scale. The log-scale pdf modelling results in Figure 1 confirm the numerical results presented in Table 3, whereby the Laplace-Rician model outperforms all reference methods utilised in this study and follows the data histograms better than the others. Figure 2 depicts a visual representation to the statistical significance values given in Table 3. Dark pixels show lower values, which also refer to better modelling results. When examining both sub-figures in Figure 2, we can easily state that for over all images, the proposed LaplaceRician model is obviously better than the state-of-the-art models.

\section{Conclusions}

In this paper, we presented a study on the statistical modelling of SAR images of various scenes. We proposed a novel statistical model, the Laplace-Rician distribution, which is based on a generalisation of the Rician distribution by replacing the Gaussian assumption with the Laplace distribution.

The performance of the Laplace-Rician in modelling SAR images corresponding to urban, agricultural, mountain, land cover, and sea surface scenes from four satellite platforms, i.e. TerraSAR-X, ALOS2, COSMO-SkyMed and Sentinel-1, was assessed in comparison to state-of-theart statistical models that include $\mathcal{K}$, Rayleigh, Rician, Weibull, lognormal and GGR [6] distributions. The results demonstrate that the proposed method achieves the best modelling results for most of the images, and outperforms state-of-the-art models for images from various frequency bands and sources.

It is interesting to note that the results show us the need for combining the advantages of non-Gaussian heavy-tailed modelling and non-zero mean reflections modelling provided by Rician model. That is quite obvious when we compare Laplace-Rician with GGR, Rician and Rayleigh directly. Furthermore, using non-zero reflections along with the heavy-tailed modelling in Laplace-Rician model shows important success in all types of SAR scenes and frequency bands. There is one specific exemption $\left(I_{25}\right)$ which contradict this contribution. Image $I_{25}$ shows the Rician is the best model, which states the need for nonzeros reflections but not heavy-tailed modelling. Moreover, There are three cases $\left(I_{5}, I_{12}\right.$ and $\left.I_{18}\right)$ that lognormal model outperforms the proposed Laplace-Rician for both of the statistical measures. These four specific examples will guide us for future works on further developments of the method on where our modelling gain is low or none.

\section{Acknowledgments}

The authors are grateful to the UK Satellite Applications Catapult for providing the COSMO-SkyMed datasets.

\section{$6 \quad$ Literature}

[1] C. Tison, J.-M. Nicolas, F. Tupin, and H. Maître, "A new statistical model for Markovian classification of urban areas in high-resolution SAR images," IEEE transactions on Geoscience and Remote Sensing, vol. 42, no. 10, pp. 2046-2057, 2004.

[2] M. E. Mejail, J. C. Jacobo-Berlles, A. C. Frery, and O. H. Bustos, "Classification of SAR images using a general and tractable multiplicative model," International Journal of Remote Sensing, vol. 24, no. 18, pp. 3565-3582, 2003.

[3] A. Achim, E. E. Kuruoglu, and J. Zerubia, "SAR image filtering based on the heavy-tailed Rayleigh model," IEEE Transactions on Image Processing, vol. 15, no. 9, pp. 2686-2693, 2006. 
Table 3 Statistical significance of SAR amplitude models for various scenes. (L-Ric: Laplace-Rician.)

\begin{tabular}{|c|c|c|c|c|c|c|c|c|c|c|c|}
\hline \multirow[b]{2}{*}{ Image } & \multirow[b]{2}{*}{ Source } & \multirow[b]{2}{*}{ Band } & \multirow[b]{2}{*}{ Scene } & \multirow{2}{*}{$\begin{array}{l}\text { Performance } \\
\text { Measures }\end{array}$} & \multicolumn{7}{|c|}{ Distribution Families } \\
\hline & & & & & $\mathrm{K}$ & Rayleigh & Rician & Weibull & Lognormal & GGR & L-Ric \\
\hline \multirow{2}{*}{$I_{1}$} & \multirow{2}{*}{ ALOS2 } & \multirow{2}{*}{$\mathrm{L}$} & & KL Div. & 0.0223 & 0.1269 & 0.1251 & 0.0109 & 0.0383 & 0.0426 & 0.0055 \\
\hline & & & Urban & KS Score & 0.0599 & 0.1623 & 0.1609 & 0.0491 & 0.0575 & 0.0966 & 0.0211 \\
\hline & & & & KL Div. & 0.0917 & 0.0858 & 0.0596 & 0.0447 & 0.0116 & 0.0707 & 0.0074 \\
\hline$I_{2}$ & Sentinel-1 & $\mathrm{C}$ & Urban & KS Score & 0.1047 & 0.0953 & 0.0776 & 0.0713 & 0.0149 & 0.0679 & 0.0165 \\
\hline & & $C$ & Urban & KL Div. & 0.0479 & 0.0426 & 0.0314 & 0.0249 & 0.0635 & 0.0351 & 0.0180 \\
\hline$I_{3}$ & Sentinel-1 & C & Urban & KS Score & 0.0703 & 0.0585 & 0.0668 & 0.0606 & 0.0719 & 0.0673 & 0.0274 \\
\hline & & & & KL Div. & 0.0875 & 0.0802 & 0.0791 & 0.0636 & 0.0248 & 0.0771 & 0.0127 \\
\hline$I_{4}$ & IerraSAR - X & $X$ & Urban & KS Score & 0.0750 & 0.0727 & 0.0809 & 0.0931 & 0.0379 & 0.0780 & 0.0342 \\
\hline & & & & KL Div. & 0.0605 & 0.0653 & 0.0798 & 0.0706 & 0.0104 & 0.2156 & 0.0230 \\
\hline$I_{5}$ & COSMO/SkyMed & $X$ & Urban & KS Score & 0.0695 & 0.0861 & 0.1174 & 0.1208 & 0.0399 & 0.1915 & 0.0533 \\
\hline & & & & KL Div. & 0.0045 & 0.1045 & 0.1038 & 0.0022 & 0.0505 & 0.0321 & 0.0044 \\
\hline$I_{6}$ & ALOS2 & $\mathrm{L}$ & Land & KS Score & 0.0186 & 0.1309 & 0.1300 & 0.0175 & 0.0643 & 0.0609 & 0.0133 \\
\hline & & & & KL Div. & 0.2660 & 0.2502 & 0.0143 & 0.0199 & 0.0274 & 0.1064 & 0.0149 \\
\hline$I_{7}$ & Sentinel-1 & $\mathrm{C}$ & Land & KS Score & 0.1805 & 0.1811 & 0.0349 & 0.0364 & 0.0394 & 0.0965 & 0.0203 \\
\hline & & & & KL Div. & 0.2567 & 0.2379 & 0.0310 & 0.0397 & 0.0251 & 0.1219 & 0.0054 \\
\hline$I_{8}$ & TerraSAR - X & $X$ & Land & KS Score & 0.1822 & 0.1808 & 0.0480 & 0.0495 & 0.0534 & 0.1069 & 0.0144 \\
\hline & & & & KL Div. & 0.0488 & 0.0987 & 0.0974 & 0.0095 & 0.0580 & 0.0098 & 0.0090 \\
\hline$I_{9}$ & TerraSAR - X & $X$ & Land & KS Score & 0.0822 & 0.1317 & 0.1302 & 0.0367 & 0.0862 & 0.0438 & 0.0190 \\
\hline & & & & KL Div. & 0.1994 & 0.1876 & 0.0411 & 0.0415 & 0.0090 & 0.1267 & 0.0072 \\
\hline$I_{10}$ & ALOS2 & $\mathrm{L}$ & Mountain & KS Score & 0.1717 & 0.1672 & 0.0690 & 0.0665 & 0.0210 & 0.1409 & 0.0133 \\
\hline & & & & KL Div. & 0.0628 & 0.0572 & 0.0300 & 0.0206 & 0.0304 & 0.0444 & 0.0022 \\
\hline$I_{11}$ & Sentinel-1 & C & Mountain & KS Score & 0.0904 & 0.0826 & 0.0679 & 0.0623 & 0.0530 & 0.0788 & 0.0178 \\
\hline & & & & KL Div. & 0.1272 & 0.1139 & 0.0610 & 0.0468 & 0.0047 & 0.0841 & 0.0139 \\
\hline$I_{12}$ & TerraSAR - X & $x$ & Mountain & KS Score & 0.1161 & 0.1065 & 0.0611 & 0.0568 & 0.0133 & 0.0711 & 0.0159 \\
\hline & & & & KL Div. & 0.0088 & 0.0105 & 0.0102 & 0.0106 & 0.0292 & 0.0107 & 0.0032 \\
\hline$I_{13}$ & ICEYE & $X$ & Mountain & KS Score & 0.0246 & 0.0332 & 0.0313 & 0.0381 & 0.0584 & 0.0349 & 0.0201 \\
\hline & & & & KL Div. & 0.2012 & 0.1836 & 0.0440 & 0.0502 & 0.1224 & 0.0898 & 0.0566 \\
\hline$I_{14}$ & ALOS2 & $\mathrm{L}$ & Agricultural & KS Score & 0.1579 & 0.1537 & 0.0491 & 0.0522 & 0.0849 & 0.0753 & 0.0430 \\
\hline & ALOS2 & $\mathrm{L}$ & Agricultural & KL Div. & 0.0134 & 0.0966 & 0.0964 & 0.0164 & 0.0218 & 0.0099 & 0.0039 \\
\hline$I_{15}$ & ALUS2 & $\mathrm{L}$ & Agricultural & KS Score & 0.0476 & 0.1428 & 0.1425 & 0.0430 & 0.0570 & 0.0300 & 0.0182 \\
\hline & & $C$ & & KL Div. & 0.1786 & 0.1693 & 0.0231 & 0.0210 & 0.0204 & 0.0805 & 0.0149 \\
\hline$I_{16}$ & Sentinel-1 & C & Agricultural & KS Score & 0.1488 & 0.1466 & 0.0428 & 0.0426 & 0.0461 & 0.0708 & 0.0190 \\
\hline$I_{17}$ & Sentinel-1 & $C$ & A oricultural & KL Div. & 0.1187 & 0.1013 & 0.0291 & 0.0242 & 0.0438 & 0.2392 & 0.0074 \\
\hline$I_{17}$ & sentinel-1 & $\mathrm{C}$ & Agricultural & KS Score & 0.1155 & 0.1079 & 0.0505 & 0.0448 & 0.0532 & 0.2365 & 0.0150 \\
\hline & & & & KL Div. & 0.3164 & 0.3040 & 0.0972 & 0.1096 & 0.0054 & 0.4446 & 0.0291 \\
\hline$I_{18}$ & TerraSAR - X & $\mathrm{X}$ & Agricultural & KS Score & 0.2084 & 0.2036 & 0.0937 & 0.0913 & 0.0208 & 0.3118 & 0.0242 \\
\hline & & & & KL Div. & 0.0120 & 0.1606 & 0.1591 & 0.0154 & 0.0170 & 0.0309 & 0.0102 \\
\hline$I_{19}$ & ALOS2 & $\mathrm{L}$ & Mixed & KS Score & 0.0479 & 0.1783 & 0.1771 & 0.0397 & 0.0622 & 0.0687 & 0.0428 \\
\hline$I_{0}$ & Sentinel-1 & $\mathrm{C}$ & Mixed & KL Div. & 0.1265 & 0.1185 & 0.0348 & 0.0305 & 0.0227 & 0.0710 & 0.0031 \\
\hline$I_{20}$ & Sentinel-1 & C & Mixed & KS Score & 0.1315 & 0.1254 & 0.0642 & 0.0592 & 0.0446 & 0.0651 & 0.0095 \\
\hline & Sentinel-1 & $\mathrm{C}$ & Mixed & KL Div. & 0.0304 & 0.0537 & 0.0534 & 0.0558 & 0.0211 & 0.0562 & 0.0057 \\
\hline$I_{21}$ & Sentinel-1 & $\mathrm{C}$ & Vixed & KS Score & 0.0435 & 0.1027 & 0.1022 & 0.0604 & 0.0205 & 0.0642 & 0.0201 \\
\hline & & $X$ & & KL Div. & 0.2966 & 0.2840 & 0.0943 & 0.1036 & 0.0168 & 0.2011 & 0.0211 \\
\hline$I_{22}$ & IerraSAR - X & $X$ & Mixed & KS Score & 0.1964 & 0.1930 & 0.1096 & 0.1115 & 0.0340 & 0.1170 & 0.0337 \\
\hline & & & & KL Div. & 0.0373 & 0.0358 & 0.0367 & 0.0279 & 0.0125 & 0.0331 & 0.0095 \\
\hline$I_{23}$ & COSMO/SkyMed & $X$ & Mixed & KS Score & 0.0450 & 0.0369 & 0.0601 & 0.0700 & 0.0289 & 0.0365 & 0.0202 \\
\hline & & & & KL Div. & 0.7297 & 0.6981 & 0.0383 & 0.1249 & 0.0106 & 0.3781 & 0.0136 \\
\hline$I_{24}$ & ALOS2 & $\mathrm{L}$ & Sea/woShips & KS Score & 0.2689 & 0.2747 & 0.0471 & 0.0808 & 0.0524 & 0.2126 & 0.0140 \\
\hline & Senti $>$ > & $\mathrm{C}$ & & KL Div. & 0.3170 & 0.2779 & 0.0215 & 0.0287 & 0.0306 & 0.1231 & 0.0254 \\
\hline$I_{25}$ & Sentinel-1 & $\mathrm{C}$ & Sea/woShips & KS Score & 0.1819 & 0.1830 & 0.0255 & 0.0338 & 0.0508 & 0.1058 & 0.0261 \\
\hline & & $C$ & & KL Div. & 0.1055 & 0.0786 & 0.0191 & 0.0126 & 0.0649 & 0.0257 & 0.0211 \\
\hline$I_{26}$ & & $\mathrm{C}$ & hips & KS Score & 0.0877 & 0.0816 & 0.0576 & 0.0513 & 0.0452 & 0.0453 & 0.0385 \\
\hline$I_{07}$ & TerraSAR - X & $x$ & Sea/woShins & KL Div. & 0.0989 & 0.0855 & 0.0491 & 0.0348 & 0.0202 & 0.0635 & 0.0118 \\
\hline$I_{27}$ & IerraSAK - X & $x$ & sea/wosnips & KS Score & 0.1039 & 0.0965 & 0.0460 & 0.0427 & 0.0363 & 0.0681 & 0.0191 \\
\hline & & & & KL Div. & 0.2449 & 0.2170 & 0.0299 & 0.0328 & 0.0199 & 0.1075 & 0.0150 \\
\hline$I_{28}$ & COSMO/SkyMed & $\mathrm{X}$ & Sea/woShips & KS Score & 0.1660 & 0.1643 & 0.0404 & 0.0386 & 0.0434 & 0.0982 & 0.0190 \\
\hline & & & & KL Div. & 0.5906 & 0.5490 & 0.0721 & 0.1409 & 0.0044 & 0.3267 & 0.0256 \\
\hline$I_{29}$ & ALOS2 & $\mathrm{L}$ & Sea/wShips & KS Score & 0.2436 & 0.2487 & 0.0730 & 0.0844 & 0.0260 & 0.1960 & 0.0228 \\
\hline$I_{2}$ & Sentinel-1 & $\mathrm{C}$ & Sea/wShins & KL Div. & 0.1701 & 0.1318 & 0.0129 & 0.0130 & 0.0618 & 0.0597 & 0.0204 \\
\hline$I_{30}$ & senunel-1 & C & sea/wsnips & KS Score & 0.1264 & 0.1205 & 0.0412 & 0.0381 & 0.0506 & 0.0734 & 0.0265 \\
\hline & & & & KL Div. & 0.1882 & 0.1610 & 0.0496 & 0.0457 & 0.0187 & 0.1045 & 0.0151 \\
\hline$I_{31}$ & COSMU/SkyMed & $X$ & Sea/wShıps & KS Score & 0.1320 & 0.1286 & 0.0565 & 0.0580 & 0.0218 & 0.0880 & 0.0114 \\
\hline & COSMO/SkvMed & $X$ & Sea/wShins & KL Div. & 0.2170 & 0.1862 & 0.0362 & 0.0384 & 0.0230 & 0.1055 & 0.0162 \\
\hline$I_{32}$ & cosinu/sкумеа & $x$ & sea/wsnips & KS Score & 0.1476 & 0.1431 & 0.0458 & 0.0483 & 0.0228 & 0.0901 & 0.0159 \\
\hline & & & & KL Div. & 0.3593 & 0.3217 & 0.0595 & 0.1049 & 0.0070 & 0.2282 & 0.0119 \\
\hline$I_{33}$ & TerraSAR - X & $X$ & Sea/wShıps & KS Score & 0.1881 & 0.1874 & 0.0615 & 0.0772 & 0.0146 & 0.1492 & 0.0105 \\
\hline
\end{tabular}




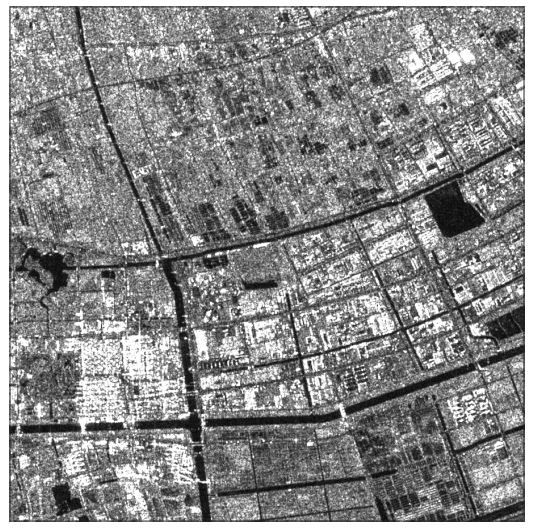

(a) $\operatorname{Urban}\left(I_{3}\right)$

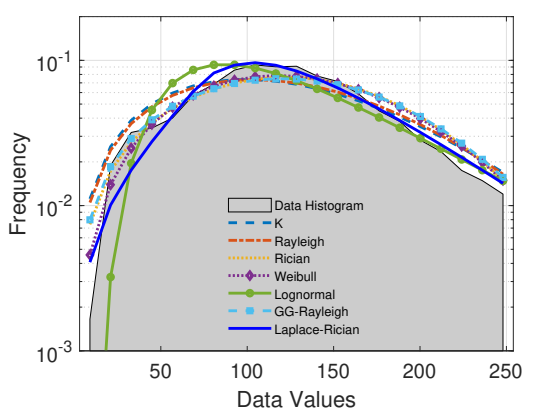

(d) Urban-logpdf

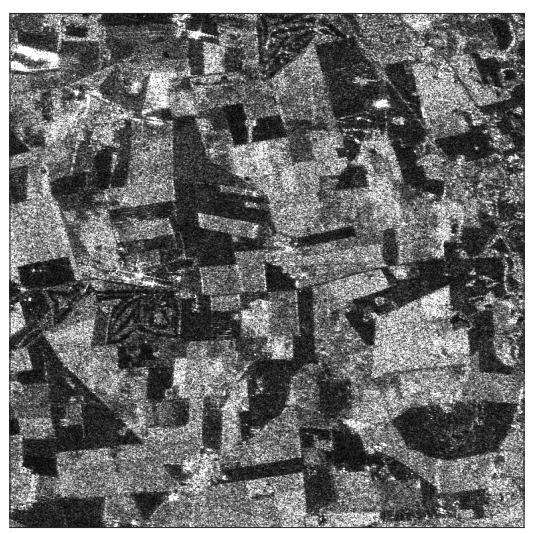

(g) Agricultural $\left(I_{15}\right)$

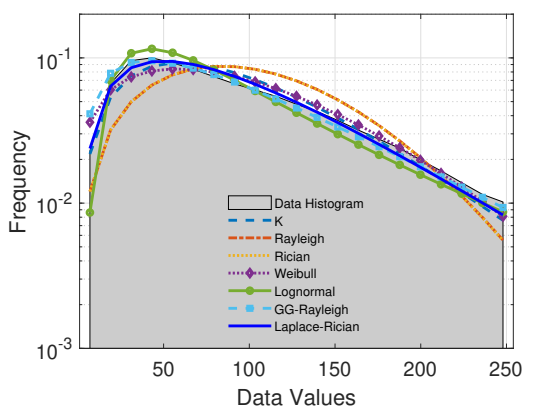

(j) Agricultural-logpdf

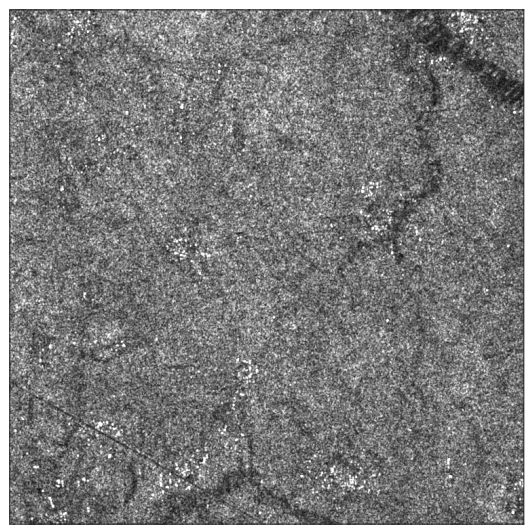

(b) Land $\left(I_{7}\right)$

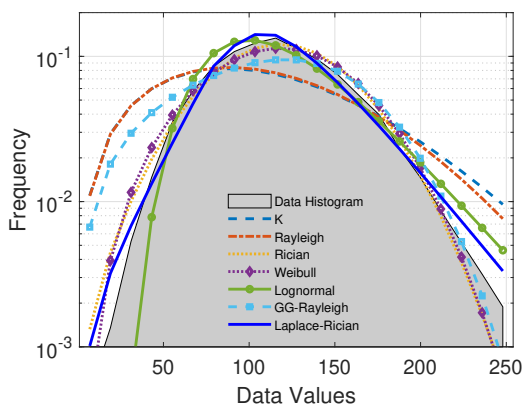

(e) Land-logpdf

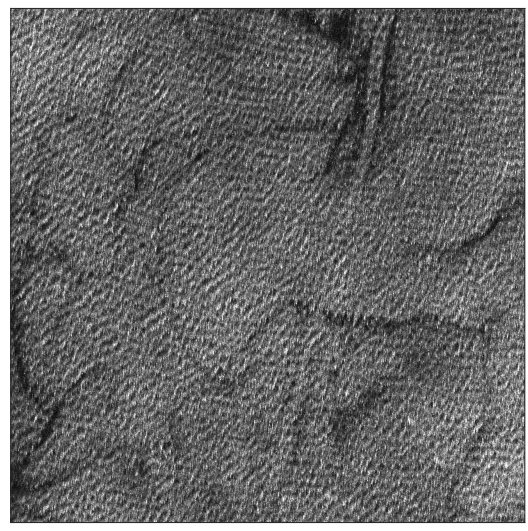

(h) Sea / woShips $\left(I_{27}\right)$

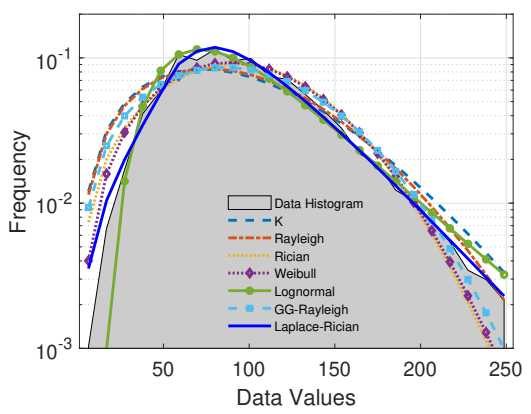

(k) Sea / woShips-logpdf

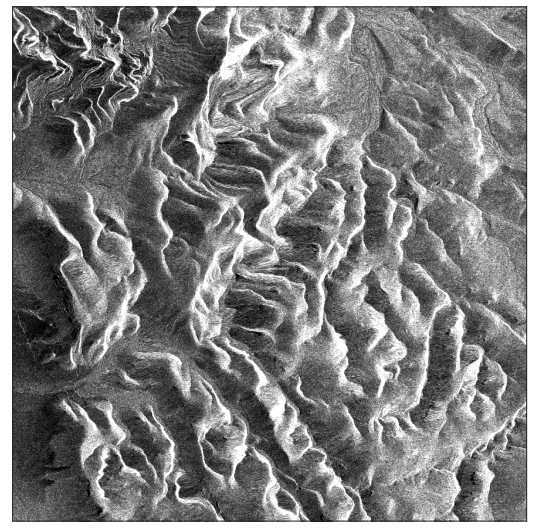

(c) Mountain $\left(I_{13}\right)$

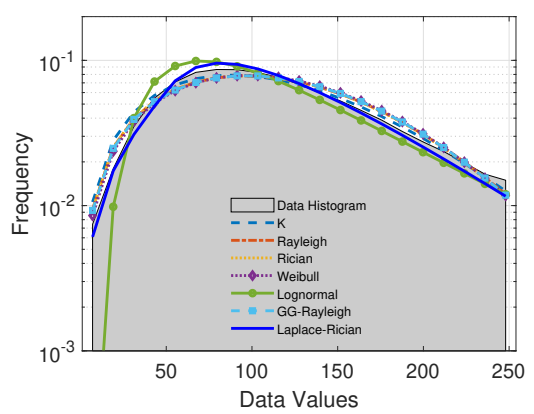

(f) Mountain-logpdf

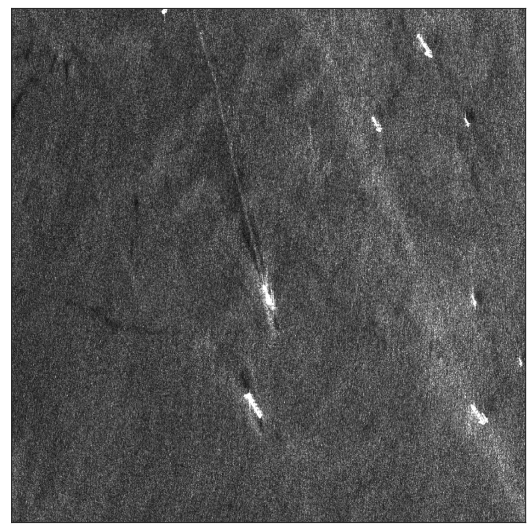

(i) Sea / wShips $\left(I_{31}\right)$

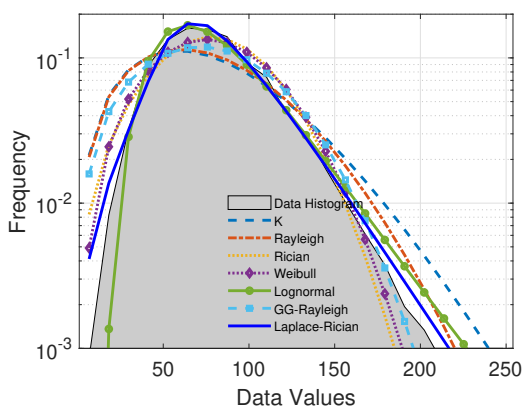

(1) Sea / wShips-logpdf

Figure 1 Subjective evaluation of SAR amplitude models.

[4] F. Argenti, A. Lapini, T. Bianchi, and L. Alparone, "A tutorial on speckle reduction in synthetic aperture radar images," IEEE Geoscience and Remote Sensing magazine, vol. 1, no. 3, pp. 6-35, 2013. 
Table 4 Modelling performance percentages in terms of KL divergence and KS score.

\begin{tabular}{lrrrc}
\hline & Rician & Weibull & Lognormal & L-Ric \\
\hline KL Div. & $12.12 \%$ & $6.06 \%$ & $21.21 \%$ & $\mathbf{6 0 . 6 1 \%}$ \\
KS Score & $3.03 \%$ & $3.03 \%$ & $12.12 \%$ & $\mathbf{8 1 . 8 2 \%}$ \\
\hline
\end{tabular}

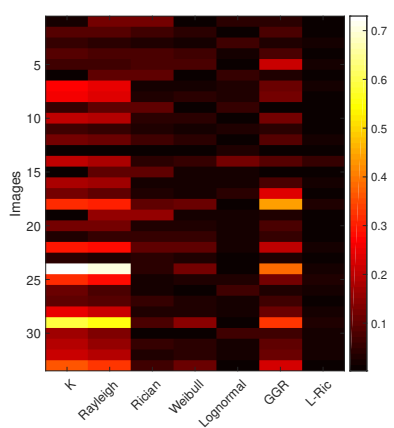

(a) KL Divergence

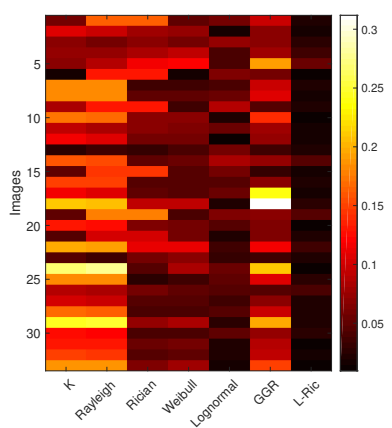

(b) KS Score
Figure 2 Statistical Significance plots.

[5] E. E. Kuruoglu and J. Zerubia, "Modeling SAR images with a generalization of the Rayleigh distribution," IEEE Transactions on Image Processing, vol. 13, no. 4, pp. 527-533, 2004.

[6] G. Moser, J. Zerubia, and S. B. Serpico, "SAR amplitude probability density function estimation based on a generalized Gaussian model," IEEE Transactions on Image Processing, vol. 15, no. 6, pp. 1429-1442, 2006.

[7] J. Sun, X. Wang, X. Yuan, Q. Zhang, C. Guan, and A. V. Babanin, "The Dependence of Sea SAR Image Distribution Parameters on Surface Wave Characteristics," Remote Sensing, vol. 10, no. 11, p. 1843,
2018.

[8] S. Chitroub, A. Houacine, and B. Sansal, "Statistical characterisation and modelling of SAR images," Signal Processing, vol. 82, no. 1, pp. 69-92, 2002.

[9] A. Yousefi, T. Liu, and G. A. Lampropoulos, "Modelling sar clutter in multi-resolution radar systems," in Photonics North 2006, vol. 6343. International Society for Optics and Photonics, 2006, p. 63432M.

[10] O. Karakuş, E. E. Kuruoğlu, and M. A. Altınkaya, "Generalized Bayesian model selection for speckle on remote sensing images," IEEE Transactions on Image Processing, vol. 28, no. 4, pp. 1748-1758, 2018.

[11] M. D. DeVore, A. D. Lanterman, and J. A. O'Sullivan, "ATR performance of a Rician model for SAR images," in Automatic Target Recognition X, vol. 4050. International Society for Optics and Photonics, 2000, pp. 34-45.

[12] T. Eltoft, "The Rician inverse Gaussian distribution: a new model for non-Rayleigh signal amplitude statistics," IEEE Transactions on Image Processing, vol. 14, no. 11, pp. 1722-1735, 2005.

[13] J. W. Goodman, "Statistical properties of laser speckle patterns," in Laser speckle and related phenomena. Springer, 1975, pp. 9-75.

[14] G. Gao, "Statistical modeling of SAR images: A survey," Sensors, vol. 10, no. 1, pp. 775-795, 2010.

[15] O. Karakuş, E. E. Kuruoğlu, and A. Achim, "Modelling sea clutter in SAR images using LaplaceRician distribution," ICASSP 2020 - IEEE International Conference on Acoustics, Speech and Signal Processing (ICASSP), 2020.

[16] H. A. Sturges, "The choice of a class interval," Journal of the American Statistical Association, vol. 21, no. 153, pp. 65-66, 1926. 